FACTA UNIVERSITATIS (NIŠ)

Ser. Math. Inform. Vol. 35, No 4 (2020), 1145-1155

https://doi.org/10.22190/FUMI2004145U

\title{
GENUINE MODIFIED BASKAKOV-DURRMEYER OPERATORS
}

\author{
Gulsum Ulusoy Ada
}

(C) 2020 by University of Niš, Serbia | Creative Commons Licence: CC BY-NC-ND

Abstract. The present paper deals with genuine Baskakov Durrmeyer operators which have preserved certain functions. We have obtained quantitative Voronovskaya and quantitative Grüss type Voronovskaya theorems using the weighted modulus of continuity. These results include the preservation properties of the classical genuine Baskakov Durrmeyer operators.

Keywords: Genuine Baskakov Durrmeyer operators; weighted modulus of continuity; Grüss Voronovskaya theorem.

\section{Introduction}

In a recent paper [22], Patel et al. considered a new construction of Baskakov operators on the unbounded interval $[0, \infty)$,

$$
V_{n}^{\vartheta}(g ; x)=\sum_{l=0}^{\infty}\left(g \circ \vartheta^{-1}\right)\left(\frac{k}{n}\right) P_{n, k}^{\vartheta}(x),
$$

where $P_{n, k}^{\vartheta}(x)=\left(\begin{array}{c}n+k-1 \\ k\end{array}\right) \frac{(\vartheta(x))^{k}}{(1+\vartheta(x))^{n+k}}, n \in \mathbb{N}, x \in[0, \infty), \vartheta$ is a continuous infinite times differentiable function satisfying the condition $\vartheta(1)=0, \vartheta(0)=0$ and $\vartheta^{\prime}(x)>$ 0 for $x \in[0, \infty)$. They investigated some direct theorems, asymptotic formula and A -statistical convergence. This function $\vartheta$ not only characterizes the operators but also characterizes the Korovkin set $\left\{1, \vartheta, \vartheta^{2}\right\}$ in a weighted function space. Inspired by this idea, many researchers studied in this direction, we can refer the readers to $[[2],[3],[4],[5],[9]$,$] .$ (1.1):

Very recently, Ada [8] have introduced Durrmeyer modifications of the operators

Received December 25, 2019; accepted March 11, 2020

2020 Mathematics Subject Classification. Primary 41A25; Secondary 41A35, 41A36 


$$
G_{n}^{\vartheta}(g ; x)=(n-1) \sum_{l=0}^{\infty} P_{n, k}^{\vartheta}(x) \int_{0}^{\infty}\left(g \circ \vartheta^{-1}\right)(u) p_{n, k}(u) d u
$$

where $p_{n, k}(u)=\left(\begin{array}{c}n+k-1 \\ k\end{array}\right) \frac{u^{k}}{(1+u)^{n+k}}$.

The operators defined in (1.2) are linear and positive. In case of $\vartheta(x)=x$, the operators in (1.2) reduce to well known Baskakov Durrmeyer operators.

Other useful modifications of positive linear operators are genuine types in approximation theory. These modifications for Bernstein durrmeyer operators were first considered by Chen [11]. Since then, many researchers have conducted studies in this field. Among the others, we refer the readers to [[10],[16],,[19],[20],[21]].

In [7], the authors introduced a genuine type modification of the operators in (1.2) defined as

$$
\begin{aligned}
D_{n}^{\vartheta}(g ; x)= & \sum_{k=1}^{\infty} P_{n, k}^{\vartheta}(x) \frac{1}{\beta(k, n+1)} \int_{0}^{\infty}\left(g \circ \vartheta^{-1}\right)(t) \frac{t^{k-1}}{(1+t)^{n+k+1}} d t \\
& +P_{n, 0}^{\vartheta}(x)\left(g \circ \vartheta^{-1}\right)(0) .
\end{aligned}
$$

In this paper, we will continue to study further approximation properties of the operators (1.3). To describe the pointwise convergence of the operators, we prove a quantitative Voronovskaya type theorem. This quantitative Voronovskaya theorem tells us the rate of pointwise convergence and an upper bound for the error of the approximation. For some other quantitative versions of Voronovskaya's theorem, we can refer the readers to [1],[13],[14].

To prove the main results, we need following moments and central moments of our new operators.

\section{Auxiliary results}

Lemma 2.1. We have

$$
\begin{gathered}
D_{n}^{\vartheta}(1 ; x)=1, D_{n}^{\vartheta}(\vartheta ; x)=\vartheta(x), \\
D_{n}^{\vartheta}\left(\vartheta^{2} ; x\right)=\frac{\vartheta^{2}(x)(n+1)+2 \vartheta(x)}{n-1}, \\
D_{n}^{\vartheta}\left(\vartheta^{3} ; x\right)=\frac{\vartheta^{3}(x)(n+1)(n+2)+6 \vartheta^{2}(x)(n+1)+6 \vartheta(x)}{(n-1)(n-2)}
\end{gathered}
$$


Lemma 2.2. If we describe the central moment operator by

$$
M_{n, m}^{\vartheta}(x)=D_{n}^{\vartheta}\left((\vartheta(t)-\vartheta(x))^{m} ; x\right)
$$

then we get

$$
\begin{gathered}
M_{n, 0}^{\vartheta}(x)=1, \quad M_{n, 1}^{\vartheta}(x)=0 \\
M_{n, 2}^{\vartheta}(x)=\frac{2 \vartheta(x)(\vartheta(x)+1)}{n-1} . \\
M_{n, 3}^{\vartheta}(x)=\frac{12 \vartheta^{3}(x)+18 \vartheta^{2}(x)+6 \vartheta(x)}{(n-1)(n-2)} \\
M_{n, 4}^{\vartheta}(x)=\frac{12\left[\vartheta^{4}(x)(n+7)+2 \vartheta^{3}(x)(n+7)+\vartheta^{2}(x)(n+9)+2 \vartheta(x)\right]}{(n-1)(n-2)(n-3)} \\
M_{n, 6}^{\vartheta}(x)=\frac{120}{(n-1)(n-2)(n-3)(n-4)(n-5)}\left[\vartheta^{6}(x) n^{2}+33 n+62\right) \\
\left.+3 \vartheta^{5}(x) n^{2}+33 n+62\right) \\
+3 \vartheta^{4}(x)\left(n^{2}+36 n+75\right) \\
+\vartheta^{3}(x)\left(n^{2}+51 n+140\right) \\
+9 \vartheta^{2}(x)(n+5) \\
+6 \vartheta(x)]
\end{gathered}
$$

for all $n, m \in \mathbb{N}$.

We suppose that:

$\left(p_{1}\right) \vartheta$ is a continuously differentiable function on $[0, \infty)$

$\left(p_{2}\right) \vartheta(0)=0, \inf _{x \in[0, \infty)} \vartheta^{\prime}(x) \geq 1$.

Let $\psi(x)=1+\vartheta^{2}(x)$ and $B_{\psi}\left(\mathbb{R}^{+}\right)=\left\{f:|f(x)| \leq M_{f} \psi(x)\right\}$, where $M_{f}$ is constant which may depend only on $f . C_{\psi}\left(\mathbb{R}^{+}\right)$denote the subspace of all continuous functions in $B_{\psi}\left(\mathbb{R}^{+}\right)$. By $C_{\psi}^{*}\left(\mathbb{R}^{+}\right)$, we denote the subspace off all functions $f \in C_{\psi}\left(\mathbb{R}^{+}\right)$for which $\lim _{x \rightarrow \infty} f(x) / \psi(x)$ is finite. Also let $U_{\psi}\left(\mathbb{R}^{+}\right)$be the space of functions $f \in C_{\psi}\left(\mathbb{R}^{+}\right)$such that $f / \psi$ is uniformly continuous. $B_{\psi}\left(\mathbb{R}^{+}\right)$is the linear normed space with the norm $\|f\|_{\psi}=\sup _{x \in \mathbb{R}^{+}}|f(x)| / \psi(x)$.

The weighted modulus of continuity defined in [17] is as follows

$$
\omega_{\vartheta}(f ; \delta)=\sup _{\substack{x, t \in \mathbb{R}^{+} \\|\vartheta(t)-\vartheta(x)| \leq \delta}} \frac{|f(t)-f(x)|}{\psi(t)+\psi(x)}
$$

for each $f \in C_{\psi}\left(\mathbb{R}^{+}\right)$and for every $\delta>0$. We observe that $\omega_{\vartheta}(f ; 0)=0$ for every $f \in C_{\psi}\left(\mathbb{R}^{+}\right)$and the function $\omega_{\vartheta}(f ; \delta)$ is nonnegative and nondecreasing with respect to $\delta$ for $f \in C_{\psi}\left(\mathbb{R}^{+}\right)$and also $\lim _{\delta \rightarrow 0} \omega_{\vartheta}(f ; \delta)=0$ for every $f \in U_{\psi}\left(\mathbb{R}^{+}\right)$. 
Lemma 2.3. ([17])For every $f \in U_{\psi}\left(\mathbb{R}^{+}\right), \lim _{\delta \rightarrow 0} \omega_{\vartheta}(f ; \delta)=0$ and

$$
|f(y)-f(x)| \leq(\psi(y)+\psi(x))\left(2+\frac{|\vartheta(y)-\vartheta(x)|}{\delta}\right) \omega_{\vartheta}(f, \delta) .
$$

Remark 2.1. If $\vartheta(x)=x$, then $\omega_{\vartheta}$ is equivalent with $\Omega_{2}$ given in [18]

$$
\Omega_{2}(f, \delta)=\sup _{\substack{x, y \geq 0 \\|h| \leq \delta}} \frac{|f(x+h)-f(x)|}{\left(1+h^{2}\right)\left(1+x^{2}\right)} .
$$

\section{Main Results}

Theorem 3.1. If the function $\vartheta$ satisfies the conditions $\left(p_{1}\right),\left(p_{2}\right)$ and $g^{\prime \prime} /\left(\vartheta^{\prime}\right)^{2}, g^{\prime} \cdot \vartheta^{\prime \prime} /\left(\vartheta^{\prime}\right)^{3} \in$ $C_{\psi}\left(\mathbb{R}^{+}\right)$, then we get for any $x \in \mathbb{R}^{+}$that

$$
\begin{aligned}
& n\left[D_{n}^{\vartheta}(g ; x)-g(x)\right]-\left(\vartheta^{2}(x)+\vartheta(x)\right) D^{2}\left(g \circ \vartheta^{-1}\right)(\vartheta(x)) \\
\leq \quad & 12\left(2+\vartheta(x)+\vartheta^{2}(x)\right)(1+\vartheta(x))^{2} \\
& \times\left\{\omega_{\vartheta}\left(\frac{g^{\prime \prime}}{\left(\vartheta^{\prime}\right)^{2}}, \delta_{n}^{\vartheta}(x)\right)+\omega_{\vartheta}\left(\frac{g^{\prime} \vartheta^{\prime \prime}}{\left(\vartheta^{\prime}\right)^{3}}, \delta_{n}^{\vartheta}(x)\right)\right\},
\end{aligned}
$$

where $\delta_{n}^{\vartheta}(x)=\left(\frac{120(1+\vartheta(x))^{6}(n+16)^{2}}{(n-5)^{4}}\right)^{\frac{1}{4}}$.

Proof. By the Taylor expansion of $g \circ \vartheta^{-1}$ we get

$$
\begin{aligned}
\left(g \circ \vartheta^{-1}\right)(\vartheta(t))= & \left(g \circ \vartheta^{-1}\right)(\vartheta(x))+D\left(g \circ \vartheta^{-1}\right)(\vartheta(x))(\vartheta(t)-\vartheta(x)) \\
& +\frac{D^{2}\left(g \circ \vartheta^{-1}\right)(\vartheta(x))(\vartheta(t)-\vartheta(x))^{2}}{2} \\
& +h(t, x)(\vartheta(t)-\vartheta(x))^{2}
\end{aligned}
$$

where

$$
h(t, x)=\frac{D^{2}\left(g \circ \vartheta^{-1}\right)(\vartheta(\epsilon))-D^{2}\left(g \circ \vartheta^{-1}\right)(\vartheta(x))}{2}
$$

and $\epsilon$ is a number between $\vartheta(x)$ and $\vartheta(t)$. We can get

$$
\begin{aligned}
& \left|D_{n}^{\vartheta}(g ; x)-g(x)-\frac{D^{2}\left(g \circ \vartheta^{-1}\right)(\vartheta(x))}{2} M_{n, 2}^{\vartheta}(x)\right| \\
\leq & D_{n}^{\vartheta}\left(|h(t, x)|(\vartheta(t)-\vartheta(x))^{2} ; x\right) .
\end{aligned}
$$


and using Lemma 2.2 we write

$$
\begin{aligned}
& \left|D_{n}^{\vartheta}(g ; x)-g(x)-\frac{2 \vartheta(x)(\vartheta(x)+1)}{n-1} \frac{D^{2}\left(g \circ \vartheta^{-1}\right)(\vartheta(x))}{2}\right| \\
\leq & D_{n}^{\vartheta}\left(|h(t, x)|(\vartheta(t)-\vartheta(x))^{2} ; x\right) .
\end{aligned}
$$

In order to complete the proof, we estimate the $D_{n}^{\vartheta}\left(|h(t, x)|(\vartheta(t)-\vartheta(x))^{2} ; x\right)$. Since

$$
\left(g \circ \vartheta^{-1}\right)^{\prime \prime}(\vartheta(t))=\frac{g^{\prime \prime}(t)}{\left(\vartheta^{\prime}(t)\right)^{2}}-g^{\prime}(t) \frac{\vartheta^{\prime \prime}(t)}{\left(\vartheta^{\prime}(t)\right)^{3}}
$$

and we have

$$
\begin{aligned}
& \frac{\left(g \circ \vartheta^{-1}\right)^{\prime \prime}(\vartheta(\epsilon))-\left(g \circ \vartheta^{-1}\right)^{\prime \prime}(\vartheta(x))}{2} \\
= & \frac{1}{2}\left\{\frac{g^{\prime \prime}(\epsilon)}{\left(\vartheta^{\prime}(\epsilon)\right)^{2}}-g^{\prime}(\epsilon) \frac{\vartheta^{\prime \prime}(\epsilon)}{\left(\vartheta^{\prime}(\epsilon)\right)^{3}}-\frac{g^{\prime \prime}(x)}{\left(\vartheta^{\prime}(x)\right)^{2}}+g^{\prime}(x) \frac{\vartheta^{\prime \prime}(x)}{\left(\vartheta^{\prime}(x)\right)^{3}}\right\} \\
= & \frac{1}{2}\left\{\frac{g^{\prime \prime}(\epsilon)}{\left(\vartheta^{\prime}(\epsilon)\right)^{2}}-\frac{g^{\prime \prime}(x)}{\left(\vartheta^{\prime}(x)\right)^{2}}+g^{\prime}(x) \frac{\vartheta^{\prime \prime}(x)}{\left(\vartheta^{\prime}(x)\right)^{3}}-g^{\prime}(\epsilon) \frac{\vartheta^{\prime \prime}(\epsilon)}{\left(\vartheta^{\prime}(\epsilon)\right)^{3}}\right\} \\
\leq & (\psi(t)+\psi(x))\left(2+\frac{|\vartheta(t)-\vartheta(x)|}{\delta}\right) \\
& \times\left\{\omega_{\vartheta}\left(\frac{g^{\prime \prime}}{\left(\vartheta^{\prime}\right)^{2}}, \delta\right)+\omega_{\vartheta}\left(\frac{g^{\prime} \vartheta^{\prime \prime}}{\left(\vartheta^{\prime}\right)^{3}}, \delta\right)\right\} .
\end{aligned}
$$

In addition, since $\psi(t)+\psi(x) \leq \delta^{2}+2 \vartheta^{2}(x)+2 \vartheta(x) \delta+2$ whenever $|\vartheta(t)-\vartheta(x)| \leq$ $\delta$, we have

$$
\begin{aligned}
|h(t, x)| \leq & 3\left(\delta^{2}+2 \vartheta^{2}(x)+2 \vartheta(x) \delta+2\right) \\
& \times\left\{\omega_{\vartheta}\left(\frac{g^{\prime \prime}}{\left(\vartheta^{\prime}\right)^{2}}, \delta\right)+\omega_{\vartheta}\left(\frac{g^{\prime} \vartheta^{\prime \prime}}{\left(\vartheta^{\prime}\right)^{3}}, \delta\right)\right\}
\end{aligned}
$$

and since $\psi(t)+\psi(x) \leq\left(\frac{\vartheta(t)-\vartheta(x)}{\delta}\right)^{2}\left(\delta^{2}+2 \vartheta^{2}(x)+2 \vartheta(x) \delta+2\right)$ whenever $|\vartheta(t)-\vartheta(x)|>$ $\delta$, we have

$$
\begin{aligned}
|h(t, x)| \leq & 3\left(\delta^{2}+2 \vartheta^{2}(x)+2 \vartheta(x) \delta+2\right) \frac{|\vartheta(t)-\vartheta(x)|^{4}}{\delta^{4}} \\
& \times\left\{\omega_{\vartheta}\left(\frac{g^{\prime \prime}}{\left(\vartheta^{\prime}\right)^{2}}, \delta\right)+\omega_{\vartheta}\left(\frac{g^{\prime} \vartheta^{\prime \prime}}{\left(\vartheta^{\prime}\right)^{3}}, \delta\right)\right\} .
\end{aligned}
$$


Choosing $\delta<1$ we deduce

$$
\begin{aligned}
|h(t, x)| \leq & 6\left(\vartheta^{2}(x)+\vartheta(x)+2\right)\left(\frac{(\vartheta(t)-\vartheta(x))^{4}}{\delta^{4}}+1\right) \\
& \times\left\{\omega_{\vartheta}\left(\frac{g^{\prime \prime}}{\left(\vartheta^{\prime}\right)^{2}}, \delta\right)+\omega_{\vartheta}\left(\frac{g^{\prime} \vartheta^{\prime \prime}}{\left(\vartheta^{\prime}\right)^{3}}, \delta\right)\right\} .
\end{aligned}
$$

Using Lemma 2.2 we have

$$
\begin{aligned}
& n\left[D_{n}^{\vartheta}(g ; x)-g(x)\right]-\left(\vartheta^{2}(x)+\vartheta(x)\right) D^{2}\left(g \circ \vartheta^{-1}\right)(\vartheta(x)) \\
& \leq \quad 6 n\left(2+\vartheta(x)+\vartheta^{2}(x)\left\{\omega_{\vartheta}\left(\frac{g^{\prime \prime}}{\left(\vartheta^{\prime}\right)^{2}}, \delta\right)+\omega_{\vartheta}\left(\frac{g^{\prime} \vartheta^{\prime \prime}}{\left(\vartheta^{\prime}\right)^{3}}, \delta\right)\right\}\right. \\
& \times M_{n, 2}^{\vartheta}(x)\left(1+\frac{1}{\delta^{4}} M_{n, 6}^{\vartheta}(x)\right) \\
& \leq \quad 6\left(2+\vartheta(x)+\vartheta^{2}(x)\right)\left\{\omega_{\vartheta}\left(\frac{g^{\prime \prime}}{\left(\vartheta^{\prime}\right)^{2}}, \delta\right)+\omega_{\vartheta}\left(\frac{g^{\prime} \vartheta^{\prime \prime}}{\left(\vartheta^{\prime}\right)^{3}}, \delta\right)\right\} \\
& \times\left\{2 \vartheta(x)(\vartheta(x)+1)+\frac{1}{\delta^{4}}\left(\frac{120(1+\vartheta(x))^{6}(n+16)^{2}}{(n-5)^{4}}\right)\right\}
\end{aligned}
$$

and if we choose $\delta_{n}^{\vartheta}=\left(\frac{120(1+\vartheta(x))^{6}(n+16)^{2}}{(n-5)^{4}}\right)^{\frac{1}{4}}$ we get

$$
\begin{array}{ll}
n & {\left[D_{n}^{\vartheta}(g ; x)-g(x)\right]-\left(\vartheta^{2}(x)+\vartheta(x)\right) D^{2}\left(g \circ \vartheta^{-1}\right)(\vartheta(x))} \\
\leq \quad & 6\left(2+\vartheta(x)+\vartheta^{2}(x)\right)\left(2 \vartheta^{2}(x)+\vartheta(x)+1\right) \\
& \times\left\{\omega_{\vartheta}\left(\frac{g^{\prime \prime}}{\left(\vartheta^{\prime}\right)^{2}}, \delta_{n}^{\vartheta}(x)\right)+\omega_{\vartheta}\left(\frac{g^{\prime} \vartheta^{\prime \prime}}{\left(\vartheta^{\prime}\right)^{3}}, \delta_{n}^{\vartheta}(x)\right)\right\} \\
\leq \quad & 12\left(2+\vartheta(x)+\vartheta^{2}(x)\right)(1+\vartheta(x))^{2} \\
& \times\left\{\omega_{\vartheta}\left(\frac{g^{\prime \prime}}{\left(\vartheta^{\prime}\right)^{2}}, \delta_{n}^{\vartheta}(x)\right)+\omega_{\vartheta}\left(\frac{g^{\prime} \vartheta^{\prime \prime}}{\left(\vartheta^{\prime}\right)^{3}}, \delta_{n}^{\vartheta}(x)\right)\right\}
\end{array}
$$

which completes the proof.

Corollary 3.1. One has the following:

1. Let $g^{\prime \prime} \in C_{\psi}\left(\mathbb{R}^{+}\right)$. The choice of $\vartheta(x)=x$ in Theorem 1 gives a quantitative Voronovskaya type theorem for $T_{n}$ which defined in [12]

$$
\left|n\left[T_{n}(g ; x)-g(x)\right]-\left(x^{2}+x\right) g^{\prime \prime}(x)\right| \leq 12(1+x)^{4} \Omega_{2}\left(g^{\prime \prime} ; \delta_{n}(x)\right)
$$

where $\delta_{n}(x)=\left(\frac{120(1+x)^{6}(n+16)^{2}}{(n-5)^{4}}\right)^{\frac{1}{4}}$. 
2. Let $g^{\prime \prime} /\left(\vartheta^{\prime}\right)^{2}, g^{\prime} \vartheta^{\prime \prime} /\left(\vartheta^{\prime}\right)^{3} \in U_{\psi}\left(\mathbb{R}^{+}\right)$. If we take limit with $n \rightarrow \infty$ in Theorem 3.1 , we get the Voronovskaya theorem for $D_{n}^{\vartheta}$

$$
\lim _{n \rightarrow \infty} n\left[D_{n}^{\vartheta}(g ; x)-g(x)\right]=\left(\vartheta^{2}(x)+\vartheta(x)\right) D^{2}\left(g \circ \vartheta^{-1}\right) \vartheta(x) .
$$

3. Let $g^{\prime \prime} /\left(\vartheta^{\prime}\right)^{2}, g^{\prime} \vartheta^{\prime \prime} /\left(\vartheta^{\prime}\right)^{3} \in U_{\psi}\left(\mathbb{R}^{+}\right)$. If $n \rightarrow \infty$ with $\vartheta(x)=x$ in Theorem 1, we obtain the Voronovskaya theorem for $T_{n}$ which defined in [12]

$$
\lim _{n \rightarrow \infty} n\left[T_{n}(g ; x)-g(x)\right]=\left(x^{2}+x\right) g^{\prime \prime}(x) .
$$

The following results is a quantitative Grüss Voronovskaya type theorems. For some applications of Grüss inequalities in approximation theory, one can refer to $[6],[15]$.

Theorem 3.2. If $g, h, \frac{g^{\prime} \vartheta^{\prime \prime}}{\left(\vartheta^{\prime}\right)^{3}}, \frac{h^{\prime} \vartheta^{\prime \prime}}{\left(\vartheta^{\prime}\right)^{3}}, \frac{g^{\prime \prime}}{\left(\vartheta^{\prime}\right)^{2}}, \frac{h^{\prime \prime}}{\left(\vartheta^{\prime}\right)^{2}} \in C_{\psi}\left(\mathbb{R}^{+}\right)$such that $\frac{(g h)^{\prime} \vartheta^{\prime \prime}}{\left(\vartheta^{\prime}\right)^{3}} \frac{(g h)^{\prime \prime} \vartheta^{\prime \prime}}{\left(\vartheta^{\prime}\right)^{2}} \in$ $C_{\psi}\left(\mathbb{R}^{+}\right)$, then we get at any point $x \in \mathbb{R}^{+}$that

$$
\begin{aligned}
& n\left|D_{n}^{\vartheta}(g h ; x)-D_{n}^{\vartheta}(g ; x) D_{n}^{\vartheta}(h ; x)-\frac{\mu_{n, 2}^{\vartheta}(x)}{\left(\vartheta^{\prime}(x)\right)^{2}}\left\{g^{\prime}(x) h^{\prime}(x)-\frac{\vartheta^{\prime \prime}(x)(g h)^{\prime}(x)}{\vartheta^{\prime}(x)}\right\}\right| \\
\leq & 12\left(2+\vartheta(x)+\vartheta^{2}(x)\right)(1+\vartheta(x))^{2} \\
& \times\left\{\omega_{\vartheta}\left(\frac{g^{\prime \prime}}{\left(\vartheta^{\prime}\right)^{2}}, \delta_{n}^{\vartheta}(x)\right)+\omega_{\vartheta}\left(\frac{g^{\prime} \vartheta^{\prime \prime}}{\left(\vartheta^{\prime}\right)^{3}}, \delta_{n}^{\vartheta}(x)\right)\right\} \\
\leq & 12\|g\|_{\psi}\left(2+\vartheta(x)+\vartheta^{2}(x)\right)(1+\vartheta(x))^{3} \\
& \times\left\{\omega_{\vartheta}\left(\frac{g^{\prime \prime}}{\left(\vartheta^{\prime}\right)^{2}}, \delta_{n}^{\vartheta}(x)\right)+\omega_{\vartheta}\left(\frac{g^{\prime} \vartheta^{\prime \prime}}{\left(\vartheta^{\prime}\right)^{3}}, \delta_{n}^{\vartheta}(x)\right)\right\} \\
& +12\|h\|_{\psi}\left(2+\vartheta(x)+\vartheta^{2}(x)\right)(1+\vartheta(x))^{3} \\
& \times\left\{\omega_{\vartheta}\left(\frac{g^{\prime \prime}}{\left(\vartheta^{\prime}\right)^{2}}, \delta_{n}^{\vartheta}(x)\right)+\omega_{\vartheta}\left(\frac{g^{\prime} \vartheta^{\prime \prime}}{\left(\vartheta^{\prime}\right)^{3}}, \delta_{n}^{\vartheta}(x)\right)\right\} \\
& +n I_{n}(g) I_{n}(h),
\end{aligned}
$$

where $I_{n}(g)=\frac{\psi(x)\left\|\left(g \circ \vartheta^{-1}\right)^{\prime \prime}\right\|_{\psi}}{2}\left(2 \mu_{n, 2}^{\vartheta}(x)+\frac{2 \vartheta(x)}{\psi(x)} \mu_{n, 3}^{\vartheta}(x)+\frac{1}{\psi(x)} \mu_{n, 4}^{\vartheta}(x)\right)$ and $I_{n}(h)$ is the analogues one.

Proof. For $x \in \mathbb{R}^{+}$and $n \in \mathbb{N}$, we have

$$
\begin{aligned}
& D_{n}^{\vartheta}(g h ; x)-D_{n}^{\vartheta}(g ; x) D_{n}^{\vartheta}(h ; x)-\mu_{n, 2}^{\vartheta}(x) \frac{g^{\prime}(x) h^{\prime}(x)}{\left(\vartheta^{\prime}(x)\right)^{2}} \\
& -\mu_{n, 2}^{\vartheta}(x) \frac{h(x) g^{\prime}(x) \vartheta^{\prime \prime}(x)}{\left(\vartheta^{\prime}(x)\right)^{3}}-\mu_{n, 2}^{\vartheta}(x) \frac{h^{\prime}(x) g(x) \vartheta^{\prime \prime}(x)}{\left(\vartheta^{\prime}(x)\right)^{3}}
\end{aligned}
$$




$$
\begin{aligned}
= & D_{n}^{\vartheta}(g h ; x)-g(x) h(x)-\frac{\mu_{n, 2}^{\vartheta}(x)}{2}\left(g h \circ \vartheta^{-1}\right)^{\prime \prime}(\vartheta(x)) \\
& -g(x)\left[D_{n}^{\vartheta}(h ; x)-h(x)-\frac{\mu_{n, 2}^{\vartheta}(x)}{2}\left(h \circ \vartheta^{-1}\right)^{\prime \prime}(\vartheta(x))\right] \\
& -h(x)\left[D_{n}^{\vartheta}(g ; x)-g(x)-\frac{\mu_{n, 2}^{\vartheta}(x)}{2}\left(g \circ \vartheta^{-1}\right)^{\prime \prime}(\vartheta(x))\right] \\
& +\left(h(x)-D_{n}^{\vartheta}(h ; x)\right)\left(D_{n}^{\vartheta}(g ; x)-g(x)\right)
\end{aligned}
$$

so using (2.5) we can write

$$
\begin{aligned}
& \left|D_{n}^{\vartheta}(g h ; x)-D_{n}^{\vartheta}(g ; x) D_{n}^{\vartheta}(h ; x)-\frac{\mu_{n, 2}^{\vartheta}(x)}{\left(\vartheta^{\prime}(x)\right)^{2}}\left\{h^{\prime}(x) g^{\prime}(x)-\frac{\vartheta^{\prime \prime}(x)(g h)(x)}{\left(\vartheta^{\prime}(x)\right)}\right\}\right| \\
\leq & \left|A_{1}\right|+\left|A_{2}\right|+\left|A_{3}\right|+\left|A_{4}\right| .
\end{aligned}
$$

By Theorem 1, we have the estimates

$$
\begin{aligned}
\left|A_{1}\right| \leq & 12\left(2+\vartheta(x)+\vartheta^{2}(x)\right)(1+\vartheta(x))^{2} \\
& \times\left\{\omega_{\vartheta}\left(\frac{g^{\prime \prime}}{\left(\vartheta^{\prime}\right)^{2}}, \delta_{n}^{\vartheta}(x)\right)+\omega_{\vartheta}\left(\frac{g^{\prime} \vartheta^{\prime \prime}}{\left(\vartheta^{\prime}\right)^{3}}, \delta_{n}^{\vartheta}(x)\right)\right\} \\
\left|A_{2}\right| \leq & 12\|g\|_{\psi}\left(2+\vartheta(x)+\vartheta^{2}(x)\right)(1+\vartheta(x))^{3} \\
& \times\left\{\omega_{\vartheta}\left(\frac{g^{\prime \prime}}{\left(\vartheta^{\prime}\right)^{2}}, \delta_{n}^{\vartheta}(x)\right)+\omega_{\vartheta}\left(\frac{g^{\prime} \vartheta^{\prime \prime}}{\left(\vartheta^{\prime}\right)^{3}}, \delta_{n}^{\vartheta}(x)\right)\right\} \\
\left|A_{3}\right| \leq & 12\|h\|_{\psi}\left(2+\vartheta(x)+\vartheta^{2}(x)\right)(1+\vartheta(x))^{3} \\
& \times\left\{\omega_{\vartheta}\left(\frac{g^{\prime \prime}}{\left(\vartheta^{\prime}\right)^{2}}, \delta_{n}^{\vartheta}(x)\right)+\omega_{\vartheta}\left(\frac{g^{\prime} \vartheta^{\prime \prime}}{\left(\vartheta^{\prime}\right)^{3}}, \delta_{n}^{\vartheta}(x)\right)\right\}
\end{aligned}
$$

In addition we can write

$$
D_{n}^{\vartheta}(g ; x)-g(x)=\left(g \circ \vartheta^{-1}\right)(\vartheta(x)) \mu_{n, 1}^{\vartheta}(x)+\frac{1}{2} D_{n}^{\vartheta}\left(\left(g \circ \vartheta^{-1}\right)^{\prime \prime}(\vartheta(\epsilon))(\vartheta(t)-\vartheta(x))^{2} ; x\right)
$$

hence we have

$$
\begin{aligned}
& \left|D_{n}^{\vartheta}(g ; x)-g(x)\right| \\
\leq & \frac{1}{2} D_{n}^{\vartheta}\left(\left|\left(g \circ \vartheta^{-1}\right)^{\prime \prime}(\epsilon)\right|(\vartheta(t)-\vartheta(x))^{2} ; x\right) \\
\leq & \left\|\left(g \circ \vartheta^{-1}\right)^{\prime \prime}\right\|_{\psi} \frac{1}{2} D_{n}^{\vartheta}\left(\left(1+\vartheta^{2}(\epsilon)\right)(\vartheta(t)-\vartheta(x))^{2} ; x\right),
\end{aligned}
$$


where $\epsilon$ is an number between $t$ and $x$. If $t<\epsilon<x$, then $1+\vartheta^{2}(\epsilon) \leq 1+\vartheta^{2}(x)$. In this case we get

$$
\left|D_{n}^{\vartheta}(g ; x)-g(x)\right| \leq \frac{\left\|\left(g \circ \vartheta^{-1}\right)^{\prime \prime}\right\|_{\psi} \psi(x)}{2} \mu_{n, 2}^{\vartheta}(x)
$$

or if $x<\epsilon<t$, then $1+\vartheta^{2}(\epsilon) \leq 1+\vartheta^{2}(t)$. In this case we get

$$
\begin{aligned}
\left|D_{n}^{\vartheta}(g ; x)-g(x)\right| & \leq \frac{\left\|\left(g \circ \vartheta^{-1}\right)^{\prime \prime}\right\|_{\psi}}{2} D_{n}^{\vartheta}\left(\left(1+\vartheta^{2}(t)\right)(\vartheta(t)-\vartheta(x))^{2} ; x\right) \\
& =\frac{\left\|\left(g \circ \vartheta^{-1}\right)^{\prime \prime}\right\|_{\psi}}{2}\left(\left(1+\vartheta^{2}(x)\right) \mu_{n, 2}^{\vartheta}(x)+2 \vartheta(x) \mu_{n, 3}^{\vartheta}(x)+\mu_{n, 4}^{\vartheta}(x)\right) .
\end{aligned}
$$

Therefore, for two cases of $\vartheta(\epsilon)$ we obtain

$$
\begin{aligned}
\left|D_{n}^{\vartheta}(g ; x)-g(x)\right| & \leq \frac{\left\|\left(g \circ \vartheta^{-1}\right)^{\prime \prime}\right\|_{\psi} \psi(x)}{2}\left\{2 \mu_{n, 2}^{\vartheta}(x)+\frac{2 \vartheta(x)}{\psi(x)} \mu_{n, 3}^{\vartheta}(x)+\frac{1}{\psi(x)} \mu_{n, 4}^{\vartheta}(x)\right\} . \\
& :=I_{n}(g)
\end{aligned}
$$

Corollary 3.2. The following hold:

1. If $g, h, g^{\prime \prime}, h^{\prime \prime} \in C_{\psi}\left(\mathbb{R}^{+}\right)$such that $(g h)^{\prime \prime} \in C_{\psi}\left(\mathbb{R}^{+}\right)$. The choice of $\vartheta(x)=x$ in Theorem 2 gives a quantitative Grüss Voronovskaya type theorem for $T_{n}$ which defined in [12]

$$
\begin{aligned}
& n\left|T_{n}(g h ; x)-T_{n}(g ; x) T_{n}(h ; x)-\left(x^{2}+x\right) g^{\prime}(x) h^{\prime}(x)\right| \\
& \leq \quad 12\left(2+x+x^{2}\right)(1+x)^{2} \Omega_{2}\left((g h)^{\prime \prime} ; \delta_{n}(x)\right) \\
&+12\|g\|_{\psi}\left(2+\vartheta(x)+\vartheta^{2}(x)\right)(1+\vartheta(x))^{3} \Omega_{2}\left(g^{\prime \prime} ; \delta_{n}(x)\right) \\
&+12\|h\|_{\psi}\left(2+\vartheta(x)+\vartheta^{2}(x)\right)(1+\vartheta(x))^{3} \Omega_{2}\left(h^{\prime \prime} ; \delta_{n}(x)\right) \\
&+n I_{n}(g) I_{n}(h) \\
& \delta_{n}(x)=\left(\frac{120(1+\vartheta(x))^{6}(n+16)^{2}}{(n-5)^{4}}\right)^{\frac{1}{4}} .
\end{aligned}
$$

2. Let $g, h, g^{\prime \prime}, h^{\prime \prime} \in U_{\psi}\left(\mathbb{R}^{+}\right)$such that $(g h)^{\prime \prime} \in U_{\psi}\left(\mathbb{R}^{+}\right)$. If $n \rightarrow \infty$ in Theorem 2, we obtain the Grüss Voronovskaya type theorem for $D_{n}^{\vartheta}$ :

$$
n\left|D_{n}^{\vartheta}(g h ; x)-D_{n}^{\vartheta}(g ; x) D_{n}^{\vartheta}(h ; x)=\frac{\left(\vartheta(x)+\vartheta^{2}(x)\right.}{\left(\vartheta^{\prime}(x)\right)^{2}}\left\{g^{\prime}(x) h^{\prime}(x)-\frac{\vartheta^{\prime \prime}(x)(g h)^{\prime}(x)}{\vartheta^{\prime}(x)}\right\}\right|
$$

3. Let $g, h, g^{\prime \prime}, h^{\prime \prime} U_{\psi}\left(\mathbb{R}^{+}\right)$such that $(g h)^{\prime \prime} \in U_{\psi}\left(\mathbb{R}^{+}\right)$. If $n \rightarrow \infty$ with we select $\vartheta(x)=x$ in Theorem 2, we get the Grüss Voronovskaya type theorem for the operators $T_{n}$ which defined in [12]:

$$
\lim _{n \rightarrow \infty} n\left|T_{n}(g h ; x)-T_{n}(g ; x) T_{n}(h ; x)=\left(x^{2}+x\right) g^{\prime}(x) h^{\prime}(x)\right| .
$$




\section{REF EREN CES}

1. T. Acar: Quantitative q-Voronovskaya and q-Grüss Voronovskaya type results for qSzasz Operators. Georgian Math. J. 23:4 (2016), 459-468.

2. T. Acar: Asymptotic Formulas for Generalized Szasz Mirakyan Operators. Applied Mathematics and Computation, 263 (2015), 223-239.

3. T. Acar, A. Aral and I. Raşa: Modified Bernstein-Durrmeyer operators. General Mathematics. 22:1 (2014), 27-41.

4. T. Acar and G. Ulusoy: Approximation properties of generalized Szasz-Durrmeyer Operators. Period. Math. Hung. 72:1 (2016), 64-75.

5. T. Acar, A. Aral, I. Rasa: Positive Linear Operators Preserving $\tau$ and $\tau^{2}$, Constructive Mathematical Analysis, 2:3 (2019), 98-102.

6. A.M. Acu, H. Gonska and I. Raşa I: Grüss type and Ostrowski type inequalities in approximation theory. Ukranian Math.J. 63:6 (2011), 843-864.

7. G. Ulusoy Ada: Better approximation of functions by genuine Baskakov Durrmeyer operators. Facta Universitatis Mathematics and Informatics. (submitted)

8. G. Ulusoy Ada: On the Generalized Baskakov Durrmeyer Operators. Sakarya University Journal of Science. 23:4 (2019), 549-553.

9. A. Aral, D. Inoan and I. Raşa: On the generalized Szasz Mirakyan operators. Results Math. 65:(3-4) (2014), 441-452.

10. M. Bodur, O.G. Yılmaz, A. Aral: Approximation by Baskakov Szasz-Stancu Operators Preserving Exponential Functions, Constructive Mathematical Analysis, 1:1 (2018), $1-8$.

11. W. Chen: On the modified Durrmeyer Bernstein operator. In: Report of the Fifth Chinese Conference on Approximation Theory, Zhen Zhou, China (1987).

12. Z. Finta: On convergence approximation theorems. J. Math. Anal. Appl. 1 (2005), 159-180.

13. S.G Gal and H. Gonska: Grüss and Grüss Voronovskaya type estimates for some Bernstein type polynomials of real and complex variables, Jaen J. Approx. 7:1 (2015), 97-122.

14. H. Gonska, P. Pitul and I. Rasa: On Peano's form of the Taylor remainder, Voronovskaja's theorem and the commutator of positive linear operators. Proc.Int. Conf. on Numerical Analysis and Approximation Theory. Cluj-Napoca, Romania, 1-24.

15. H. Gonska and G. Tachev: Grüss type inequalities for positive linear operators with second order moduli. Mathematica Vesnik. 63:4 (2011), 47-252.

16. V. Gupta and N. Malik: Genuine link Baskakov Durrmeyer operators. Georgian Math. J. 2016.

17. A. Holhos: Quantitative estimates for positive linear operators in weighted space. General Math. 16:4 (2008), 99-110.

18. N. Ispir: On modifid Baskakov Operators on weighted spaces. Turk J. Math. 25:3 (2001), 355-365.

19. A. Kajla: On the Bezier Variant of the Srivastava-Gupta Operators, Constructive Mathematical Analysis. 1:2 (2018), 99-107.

20. S.A Mohiuddine, T. Acar and M.A. Alghamid: Genuine modified Benstein Durrmeyer operators. Journal of Inequalities and Applications, 1 (2018), 104. 
21. F. Ozsarac, T. Acar: Reconstruction of Baskakov operators preserving some exponential functions, Mathematical Methods in the Applied Sciences, 42:16, (2019), 51245132 .

22. P. Patel, V.N. Mishra and M. Örkcü: Some approximation properties of the generalized Baskakov operators. Journal of Interdisciplinary Mathematics. 21:3 (2018), 611-622.

\author{
Gulsum Ulusoy ADA \\ Faculty of Science \\ Department of Mathematics \\ Çankırı18000 \\ Turkey \\ ulusoygulsum@hotmail.com
}

